\title{
Barriers to continuous professional development participation for radiographers in Kenya
}

\author{
${ }^{1}$ Department of Medical Imaging Sciences, Faculty of Diagnostic Sciences, Kenya Medical Training College, Nairobi Campus, Kenya \\ ${ }^{2}$ Department of Medical Imaging and Radiation Sciences, Faculty of Health Sciences, University of Johannesburg, South Africa \\ ${ }^{3}$ Department of Medical Imaging Sciences, Faculty of Diagnostic Sciences, Kenya Medical Training College, Nyeri Campus, Kenya
}

L G Kanamu, ${ }_{1}^{1}$ MTech Rad, BTech Rad (D), HDip Med Ed, Dip Rad (D); B van Dyk, ${ }^{2}$ MTech Rad, BTech Rad (U/S), NDip Rad (T), NDip Rad (D); L Chipeya, ${ }^{2}$ MPhil HE, BAdmin Hons Ind Psych, BAdmin, NHD (NM), NDip Rad (D \& NM); S N Kilaha, ${ }^{3}$ PhD, MMed Ed, HDip Med Ed, Dip Rad (D)

Corresponding author: B van Dyk (bvandyk@uj.ac.za)

\begin{abstract}
Background. Updating knowledge and skills on an ongoing basis is an important requirement if one is to remain professionally relevant. Formalised continuous professional development (CPD) is, therefore, essential to stay up to date in a dynamic work environment. The majority of radiographers in Kenya work in remote rural health facilities, where CPD activities are limited. The question therefore arose: 'To what extent are radiographers participating in CPD activities and what constitute barriers to participation?'

Objective. To describe the challenges that affect diagnostic radiographers' participation in CPD activities in Kenya.

Methods. The study targeted radiographers who were registered with the Society of Radiography in Kenya (SORK). Two hundred and fifty prospective participants were recruited from the SORK database, using the fish-bowl sampling method. Questionnaires with self-addressed stamped envelopes were posted by ordinary mail to facilitate ease of return, while telephonic follow-up improved the response rate.

Results. The study revealed that $69 \%$ of diagnostic radiographers in Kenya were effectively participating in CPD activities. Barriers to CPD participation included time constraints (62\%), financial constraints (66\%), lack of information (54\%), organisational culture (47\%), paucity of resources (58\%), and difficulty in being selected by their organisation to attend CPD activities (42\%).

Conclusion. Professional development in a rapidly developing and expanding profession such as radiography is critical for best practice. Although the majority of diagnostic radiographers in Kenya participate in CPD, a large number do not owing to various challenges. A lack of finances was one of the most significant factors that served as a barrier. SORK, employers and institutions of higher education all have a responsibility towards the culture of lifelong learning. As the professional organisation representing radiographers, SORK should engage all stakeholders to collectively address the barriers to CPD participation for radiographers in Kenya.
\end{abstract}

Afr J Health Professions Educ 2017;9(1):17-20. DOI:10.7196/AJHPE.2017.v9i1.605

Obtaining a professional qualification can be viewed as a starting point that demonstrates the minimum level of competence within a given field of study. ${ }^{[1]}$ The half-life of knowledge varies between 2 and 5 years. Therefore, knowledge obtained at the point of professional qualification is insufficient to support an individual throughout a lifetime of professional practice. New learning opportunities that translate into advanced professional competencies should hence be created ${ }^{[2]}$ Professional practitioners should consequently improve their knowledge and skills to stay abreast and meet the demands of the dynamic environment in which they operate.

Continuous professional development (CPD) evolved from the need for a platform that allows professionals to become lifelong learners and stay up to date with new knowledge, developments and skills. CPD can be defined as 'the continuous and systematic maintenance, improvement and broadening of knowledge, expertise and skills for the execution of professional duties throughout the practitioners' working life. ${ }^{[3]} \mathrm{CPD}$ is, therefore, an ongoing process, encompassing formal and informal education, which builds on an initial professional qualification and addresses the learning needs of practitioners in preparation for new responsibilities or extended roles.

After the implementation in 2004 of compulsory CPD for Kenyan health professionals, the Society of Radiography in Kenya (SORK) established CPD guidelines for radiography, in which they undertook to:

- ensure that $\mathrm{CPD}$ addresses the short-term learning needs of radiographers, while maintaining high competence standards for the profession in the longer term

- encourage radiographers to participate in CPD by providing a systematic framework that supports lifelong learning

- accredit all CPD activities

- promote CPD by working in partnership with employers, academic institutions, government agencies and other relevant bodies.

The current CPD requirement for radiographers in Kenya is based on the attainment of 40 credits obtained over a 2 -year cycle. Numerous factors may influence CPD participation among members of a profession. These factors can include professional attitude, work pressure, job satisfaction, organisational culture, dissemination of information, financial or time constraints, or lack of resources, including access to journals or other educational materials. Some of these factors are especially detrimental to professionals in rural and remote work environments. ${ }^{[4]}$ As the majority of radiographers (63.6\%) work in rural and remote health facilities in Kenya, this study was conducted to investigate the barriers experienced by diagnostic radiographers to CPD participation. 


\section{Methods}

This was an empirical study; it therefore relied on perceptions, experiences and observations of participants. A quantitative descriptive survey allowed for the statistical comparison between variables.

\section{Population and sampling}

The population comprised $\sim 1000$ diagnostic radiographers, who were registered as members of SORK at the time of the study. To calculate the sample size, a confidence level of $95 \%$ with a confidence interval (CI) of $5 \%$ was set by using the following formula: $N=P(100 \%-P) /(\mathrm{SE})^{2}$. $(N=$ calculated sample size (296); $P$ = proportion of radiographers (74\%) expected to participate in CPD activities, as derived from responses in the pilot study $(N=19)$; $\mathrm{SE}=$ standard error, calculated by dividing the CI by $1.96(5 / 1.96=2.55).)^{[5]}$

A calculated sample size of 296 participants was ideal, but owing to financial constraints, only 250 questionnaires were dispatched to radiographers, who were randomly selected from the SORK database, using the fish-bowl technique. ${ }^{[6]}$

\section{Data collection}

The dependent variable was defined as CPD participation, while the independent variables consisted of a number of factors with the ability to influence participation rate, as identified from the literature. ${ }^{[4]}$ Data were collected with the aid of a self-administered questionnaire.

Content validity of the questionnaire was addressed by providing a set of questions as derived by an extensive literature review. ${ }^{[4]}$ External consistency was pretested by subjecting the questionnaire to a pilot study, allowing for information that was not clearly understood to be addressed before data collection commenced. The pilot study thus measured the reliability of the instrument.

Questionnaires, including an explanatory letter, were mailed to prospective participants with a stamped self-addressed envelope to facilitate ease of return. Questionnaire items targeted information regarding barriers to CPD participation, while questionnaires were completed anonymously.

\section{Statistical analysis}

Data analysis was performed using SPSS version 14.0 (SPSS Inc., USA). While descriptive analysis aimed to describe the sample and summarise variables, cross tabulations were employed to demonstrate relationships between variables. Pearson's $\chi^{2}$ test was likewise employed to compare variances between categorical data in non $-2 \times 2$ tables, followed by Cramér's $V$ test, used as a post test to determine the strength of the association, with a value between 0 and 1. (Low association $<0.3$; moderate association $0.3-0.5$; high association $>0.50$. ${ }^{[6]} \mathrm{A} p$-value of $<0.05$ indicated that a relationship exists between two variables and the null hypothesis was rejected.

\section{Ethical considerations}

Participants were safeguarded against ethical misconduct by obtaining permission to collect data from the National Council for Science and Technology (NCST/5/002/R/537/5) in the Ministry of Higher Education, Science and Technology of the government of Kenya. The research was further approved by the Higher Degrees and Research Ethics Committees of the University of Johannesburg, South Africa (ref. no. HDC 40/2009). A letter explaining the purpose of the study and giving assurance of anonymity and voluntary participation, accompanied the questionnaires.

\section{Results}

Of the 250 questionnaires, 162 (65\%) were returned and available for analysis. Table 1 indicates that just over two-thirds (69\%) of diagnostic radiographers, registered with SORK, were enrolled in a CPD programme.

Factors influencing CPD participation in the Kenyan context are displayed in Fig. 1. Financial constraints, time constraints and a paucity of resources were rated as the most significant barriers to CPD participation among radiographers.

Tables 2 and 3 show the extent to which each of the factors in Fig. 1 affected participation in CPD activities. The factors indicate the options selected by each participant in response to a Likert scale.

The lack of finances, as indicated in Table 3, mostly affected participants' involvement in formal training programmes ( $p=0.017$; Cramér's $V=0.302$ ), the acquisition of $(p=0.048$; phi $=0.267)$ and reading $(p=0.036$; Cramér's $\mathrm{V}=0.258)$ of scientific journals.

Although time constraints $(p=0.576)$, organisational culture $(p=0.341)$, availability of resources $(p=0.46)$ and difficulty in getting nominated/ selected by the organisation to attend CPD activities $(p=0.226)$ affected CPD participation to some extent, these factors showed no statistical significance.

Dissemination of CPD information to SORK members appeared to be the least significant barrier. However, statistical significance with a weak association was recorded between communication from SORK to its members and the regularity with which seminars or workshops were attended.

\section{Discussion}

Knowledge and skills become obsolete in the absence of efforts to update them on an ongoing basis. ${ }^{[7]}$ However, this study found that diagnostic

\begin{tabular}{ll} 
Table 1. Overall participation in CPD activities & \\
\hline Category & $\boldsymbol{n}(\%)$ \\
\hline Are you enrolled in a CPD programme $(N=162) ?$ & \\
$\quad$ Yes & $112(69)$ \\
$\quad$ No & $49(30)$ \\
$\quad$ No response & $1(1)$ \\
Do you work in a rural or urban setting $(N=162) ?$ & \\
$\quad$ Urban & $59(36)$ \\
$\quad$ Rural & $103(64)$
\end{tabular}

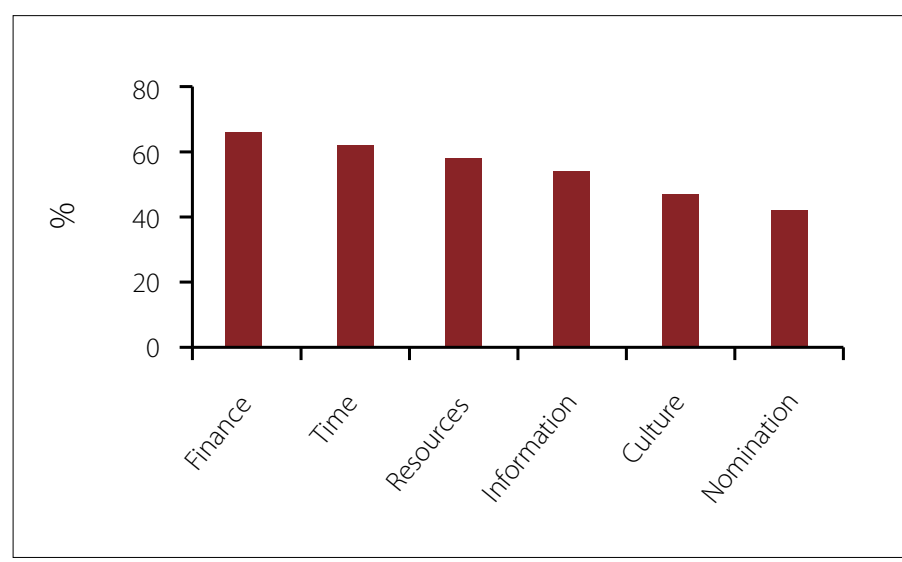

Fig. 1. Barriers to CPD participation among diagnostic radiographers in Kenya. 


\begin{tabular}{|c|c|c|c|c|c|c|}
\hline $\begin{array}{l}\text { Scale used to rate the extent } \\
\text { to which CPD participation is } \\
\text { affected by each factor }\end{array}$ & $\begin{array}{l}\text { Financial } \\
\text { constraints, \% }\end{array}$ & $\begin{array}{l}\text { Time } \\
\text { constraints, \% }\end{array}$ & $\begin{array}{l}\text { Paucity of } \\
\text { resources, \% }\end{array}$ & $\begin{array}{l}\text { Organisational } \\
\text { culture, \% }\end{array}$ & $\begin{array}{l}\text { Lack of } \\
\text { information, \% }\end{array}$ & $\begin{array}{l}\text { Difficulty in getting } \\
\text { nominated or selected to } \\
\text { attend CPD activities, \% } \\
\end{array}$ \\
\hline Not affected (0) & 3 & 7 & 7 & 20 & 15 & 27 \\
\hline Least extent (minimal chance) (1) & 7 & 9 & 12 & 14 & 24 & 12 \\
\hline Some extent (some chance) (2) & 25 & 30 & 29 & 25 & 20 & 15 \\
\hline Great extent (3) & 34 & 23 & 17 & 8 & 10 & 15 \\
\hline No response & 31 & 31 & 35 & 33 & 31 & 31 \\
\hline Total & 100 & 100 & 100 & 100 & 100 & 100 \\
\hline
\end{tabular}

Table 3. Cross tabulations between barriers and CPD participation $(N=112)$

\begin{tabular}{lll}
\hline Test & Value & $p$-value \\
\hline
\end{tabular}

Effect of financial constraints on CPD participation

1 . Were you enrolled in any formal train-

ing programme in the last 12 months?

$\begin{array}{lll}\text { Pearson's } \chi^{2} & 10.240 & 0.017 \\ \text { Cramér's V } & 0.302 & \text { Moderate association }\end{array}$

2. How often do you read scientific journals?

$\begin{array}{lll}\text { Pearson's } \chi^{2} & 22.117 & 0.036 \\ \text { Cramér's V } & 0.258 & \text { Weak association }\end{array}$

3. Do you subscribe to scientific

journals?

$\begin{array}{lll}\text { Pearson's } \chi^{2} & 7.894 & 0.048 \\ \text { Cramér's V } & 0.267 & \text { Weak association }\end{array}$

Effect of lack of information on CPD participation at seminars and workshops

1. How regularly do you attend

seminars/workshops?

$\begin{array}{lll}\text { Pearson's } \chi^{2} & 18.745 & 0.027 \\ \text { Cramér's V } & 0.238 & \text { Weak association }\end{array}$

2. How often do you read scientific journals?

$\begin{array}{lll}\text { Pearson's } \chi^{2} & 18.745 & 0.027 \\ \text { Cramér's V } & 0.238 & \text { Weak association }\end{array}$

radiographers experienced barriers to CPD participation, which need to be addressed.

\section{Financial constraints}

A large proportion of radiographers in Kenya work in health facilities in rural areas. Participants in rural areas generally experience additional infrastructure challenges, such as poorly maintained transport networks, which increase the cost of CPD participation outside their work stations. ${ }^{[8]}$ This may explain why only $34 \%$ were not affected by financial constraints.

Although employers could provide financial support, managers rarely prioritise the need for CPD when allocating resources. ${ }^{[9]}$ As salaries are inadequate, a good incentive or remuneration policy can act as a catalyst for the improvement and broadening of knowledge and skills. ${ }^{[10]}$ This can be achieved by linking remuneration and promotion to minimum educational standards and experience for each professional level. Professionals will, therefore, strive to acquire the qualifications and/or competencies required to advance professionally. Although not applicable to all radiographers, the remuneration policy in the Kenyan public sector makes provision for the acquisition of additional qualifications or specialisation to enhance work performance and facilitate advancement within the profession. ${ }^{[1]}$ It, therefore, seems as if the relatively low salaries prevent radiographers from furthering their studies or subscribing to expensive scientific journals, when they are personally being held responsible for the financial implications.

\section{Time constraints}

Lack of time to attend CPD activities affected $62 \%$ of the diagnostic radiographers in this study. Although not investigated in our study, work pressure and family responsibilities were previously found to have an adverse effect on CPD participation. ${ }^{[12]}$

\section{Paucity of resources}

Most respondents in our study (58\%) were negatively affected by a limitation of resources. Lack of access to resources contributes greatly to the challenges facing professionals. This is more obvious in rural settings, where access to resources, such as scientific journals, professional materials, the internet and study clubs, are limited.

\section{Lack of information and communication}

Competency cannot be separated from effective communication, as good personal relations and communication of information provide a stimulus that enhances participation in $\mathrm{CPD}^{\left[{ }^{[13]}\right.}$ Although the communication between SORK and its members seems to be good, a lack of information still affects the rate at which radiographers in Kenya attend seminars or workshops.

As the professional body representing radiographers in Kenya, SORK has a key role in supporting the education, lifelong learning and professional development of its members. We are, therefore, of the opinion that SORK should support members to engage in CPD by creating a platform where diagnostic radiographers can share scientific knowledge - a journal would be ideal for this purpose. ${ }^{[3,15]}$ Furthermore, communication with members can be improved by providing the most current information regarding CPD activities on the website and exploring the internet as a means of providing a range of CPD opportunities. ${ }^{[3,15]}$ Apart from arranging regular seminars, SORK can partner with tertiary institutions or structure the content of CPD activities to address key educational issues to improve professional practice, knowledge, skills and attitudes of radiographers. ${ }^{[3,15]}$ By formulating a CPD policy employers can, additionally, be guided in developing an organisational culture conducive to participation. ${ }^{[8,15]}$ 


\section{Organisational culture}

Although organisational culture showed no statistically significant association with CPD participation in this study, it is generally believed to contribute to non-participation of diagnostic radiographers in CPD. Professionals who work in a supportive environment maintain their motivation and job satisfaction. ${ }^{[14]}$ Employers are, therefore, responsible to ensure that their workforce maintain appropriate standards by adopting a pro-CPD culture. ${ }^{[3]}$

In our study, only $20 \%$ of participants expressed satisfaction with their organisational culture towards CPD (Table 1). This implies that service delivery may be adversely affected, as professionals lack opportunities to update their professional knowledge and skills. Organisational support could be achieved by advocating a culture that encourages employees to maintain and improve their professional knowledge and skills for optimal service delivery and personal advancement. ${ }^{[15]}$ Support may be in the form of funding or subsidising of formal or informal CPD activities, ${ }^{[8]}$ or by providing learning resources in the workplace, inclusive of current and relevant scientific publications and internet access. ${ }^{[3,15]}$

\section{Difficulty in getting nominated or selected to attend CPD activities}

In our study, $42 \%$ of participants encountered difficulties in getting nominated by their employers to attend CPD activities (Fig. 1). It seems easier to obtain approval to enrol in distance learning programmes than in formal face-to-face programmes. Although not statistically significant $(p=0.226$ ), radiographers might feel deprived of formal learning opportunities owing to a lack of goodwill from their employers.

\section{Study limitations}

As all legally practising radiographers are not registered members of SORK, the sample was restricted to include SORK members only. The organisation currently has no power to compel radiographers to become members owing to the lack of a legislative framework.

\section{Conclusion}

Professional development in a rapidly developing and expanding profession such as medical radiography is critical for best practice. Although the majority of diagnostic radiographers in Kenya participate in CPD, a large number do not participate owing to various challenges (Fig. 1).

While organisations need to support CPD activities to optimise the benefit to the organisation, it is problematic that some still value $\mathrm{CPD}$ as a cost and not an investment. Although the majority of radiographers in our study considered CPD as an investment, the associated cost made it difficult for them to fully engage in CPD activities, which may have a negative impact on the standard of radiological services in Kenya.

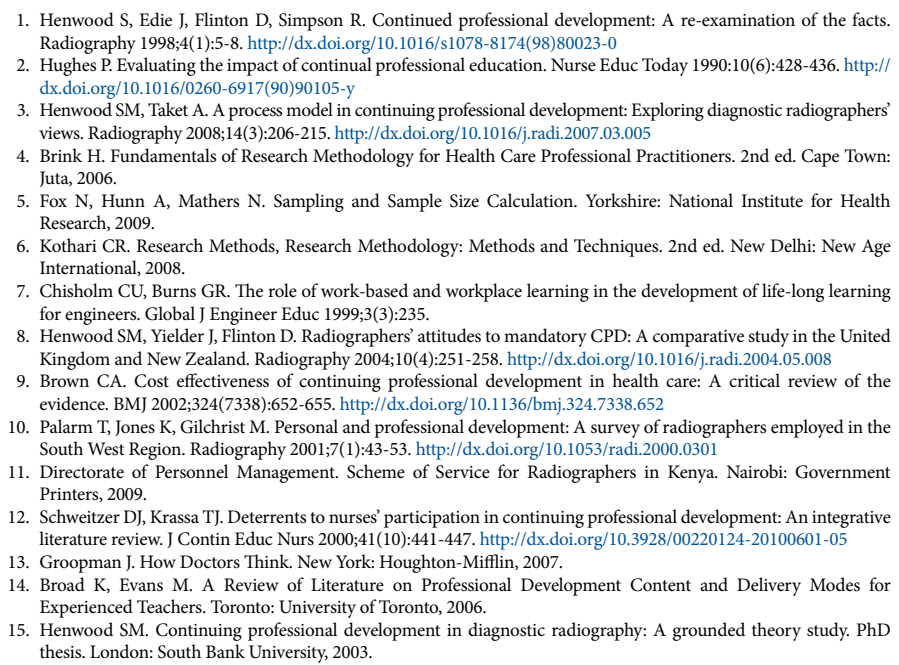

15. Henwood SM. Continuing professional development in diagnostic radiography: A grounded theory study. PhD thesis. London: South Bank University, 2003. 